OPEN ACCESS

Edited by:

Nuria Salazar,

Spanish National Research Counci (CSIC), Spain

Reviewed by:

Guijie Chen,

Nanjing Agricultural University, China

Rosaura Leis,

University of Santiago de

Compostela, Spain

*Correspondence:

Raylene A. Reimer

reimer@ucalgary.ca

Specialty section

This article was submitted to

Nutrition and Microbes,

a section of the journa

Frontiers in Nutrition

Received: 15 October 2021 Accepted: 13 December 2021

Published: 14 January 2022

Citation:

Wang W, Nettleton JE, Gänzle MG and Reimer RA (2022) A

Metagenomics Investigation of

Intergenerational Effects of

Non-nutritive Sweeteners on Gut Microbiome. Front. Nutr. 8:795848.

doi: 10.3389/fnut.2021.795848

\section{A Metagenomics Investigation of Intergenerational Effects of Non-nutritive Sweeteners on Gut Microbiome}

\author{
Weilan Wang ${ }^{1}$, Jodi E. Nettleton ${ }^{2}$, Michael G. Gänzle ${ }^{3}$ and Raylene A. Reimer ${ }^{1,4 *}$ \\ ${ }^{1}$ Faculty of Kinesiology, University of Calgary, Calgary, AB, Canada, ${ }^{2}$ WK Health Centre, Division of Gastroenterology and \\ Nutrition, Halifax, NS, Canada, ${ }^{3}$ Department of Agricultural, Food and Nutritional Science, University of Alberta, Edmonton, \\ $A B$, Canada, ${ }^{4}$ Department of Biochemistry and Molecular Biology, Cumming School of Medicine, University of Calgary, \\ Calgary, $A B$, Canada
}

To identify possible mechanisms by which maternal consumption of non-nutritive sweeteners increases obesity risk in offspring, we reconstructed the major alterations in the cecal microbiome of 3-week-old offspring of obese dams consuming high fat/sucrose (HFS) diet with or without aspartame (5-7 mg/kg/day) or stevia (2-3 mg/kg/day) by shotgun metagenomic sequencing $(n=36)$. High throughput $16 \mathrm{~S}$ rRNA gene sequencing ( $n=105$ ) was performed for dams, 3- and 18-week-old offspring. Maternal consumption of sweeteners altered cecal microbial composition and metabolism of propionate/lactate in their offspring. Offspring daily body weight gain, liver weight and body fat were positively correlated to the relative abundance of key microbes and enzymes involved in succinate/propionate production while negatively correlated to that of lactose degradation and lactate production. The altered propionate/lactate production in the cecum of weanlings from aspartame and stevia consuming dams implicates an altered ratio of dietary carbohydrate digestion, mainly lactose, in the small intestine vs. microbial fermentation in the large intestine. The reconstructed microbiome alterations could explain increased offspring body weight and body fat. This study demonstrates that intense sweet tastants have a lasting and intergenerational effect on gut microbiota, microbial metabolites and host health.

Keywords: non-nutritive sweeteners, metagenomic construction, gut microbiome, maternal diet, obesity risk

\section{INTRODUCTION}

Low-calorie sweeteners, also known as non-nutritive sweeteners, have been used for decades to replace sugar and reduce the caloric content of foods and beverages while maintaining the sweet taste (1). The consumption of non-nutritive sweeteners has raised concerns about potential detrimental effects of long-term intake which have been demonstrated in some rodent studies $(2-5)$ but less consistently in human studies $(4,6-8)$. Aspartame and stevia are two commonly used low-calorie sweeteners whose metabolism has been investigated in different species (3, 914). Aspartame is a dipeptide-methyl ester (4) which, upon ingestion, is rapidly metabolized into methanol, aspartate and phenylalanine and absorbed into the systemic circulation $(4,13)$. Rebaudioside A, the stevia glycoside used in this study, is resistant to hydrolysis by pancreatic or brush border enzymes but is converted to the aglycone steviol by ileal or colonic bacteria expressing 
$\beta$-glucosidases; absorption and metabolism of steviol in the liver occurs in a similar manner in humans and rats $(11,12,15)$. Bacteroides isolates from human microbiota were identified as the most efficient bacteria in hydrolyzing rebaudioside $\mathrm{A}$ to steviol (12).

Non-nutritive sweeteners are non-toxic to human adults (3, 9, 11) but detrimental effects, including disrupted gut microbiota, impaired glucose homeostasis and higher risk of obesity have been observed in offspring of rodents consuming non-nutritive sweeteners and infants of pregnant women consuming beverages with non-nutritive sweeteners $(2,3,5,7,9)$. We recently reported that maternal consumption of aspartame and stevia altered the expression of genes related to the mesolimbic reward system in 3 -week and 18-week old rat offspring, and altered gut microbiota in the 3-week old offspring (9). Both changes could plausibly explain the associations between maternal aspartame and stevia consumption with the higher body weight gain and body fat of their offspring (9). The former could promote higher food intake while the latter is related to the energy derived from intestinal absorption of nutrients and microbial metabolites. At the same time, we transplanted fecal microbiota of the offspring from aspartame and stevia consuming dams to germ free mice, which resulted in an increase in glucose intolerance, body weight gain and body fat of the recipient mice (9). Despite the compositional changes detected in this study and the convincing transfer of the obese phenotype into germ free mice through fecal microbiota transplant, our understanding of the functional changes that occurred in the offspring microbiome in response to maternal consumption of low-calorie sweeteners remains limited.

Therefore, the objective of the present study was to reconstruct the major alterations in the cecal microbiome of 3-week old offspring that are related to maternal consumption of aspartame or stevia during pregnancy and lactation. Short chain fatty acids (SCFA), the end products of gut microbial fermentation, were selected as the most direct mediator to investigate microbial-associated energy metabolism (16). We use shotgun metagenomics and $16 \mathrm{~S}$ rRNA gene sequencing to elucidate the connections between key microbes, metabolic functions, and the physiological outcomes of offspring, with the goal of identifying the possible mechanisms by which maternal aspartame and stevia consumption exert effects on offspring that never directly consumed the sweeteners themselves.

\section{MATERIALS AND METHODS}

\section{Animals and Samples}

Ethical approval was granted by the University of Calgary Animal Care Committee (Protocol\#AC15-0079) and conformed to the Guide to the Care and Use of Experimental Animals. Obesity was induced in 8-week-old female Sprague-Dawley rats using a 10 -week feeding period with a high fat/high sucrose diet (HFS, $39 \%$ fat and $44 \%$ sucrose, Dyets \#102412), the composition of which has been previously published (17). Given that SpragueDawley rats can be obesity-prone or obesity-resistant when fed a HFS diet (18), we chose the top $n=45$ best weight gainers from the $n=135$ rats included in the diet-induced obesity protocol (Figure 1). The selected obese female rats were bred with male Sprague-Dawley rats and randomly allocated to one of three groups of $n=15$ each throughout pregnancy and lactation: (1) WTR (HFS + Water); (2) APM (HFS + aspartame) (5-7 mg/kg, Fluka, Ottawa, ON, Canada); (3) STV (HFS + stevia) (2-3 mg/kg Rebaudioside A, Sigma-Aldrich, Oakville, $\mathrm{ON}$, Canada). Aspartame and stevia were administered in the drinking water as described (9). These doses were selected within the acceptable daily intake (aspartame: $40 \mathrm{mg} / \mathrm{kg}$, stevia: $4 \mathrm{mg} / \mathrm{kg}$ ) (19) established by the Food Directorate of Health Canada and reflect physiological daily intake in humans (20). To minimize the effects of varying litter size, litters were culled to 10 offspring ( 5 females +5 males) at birth. Offspring were weaned at 3 weeks of age and fed a control diet (AIN-93, Dyets, Bethlehem, PA, USA) until 18 weeks of age. Rats were fed ad libitum and housed in a temperature- and humidity-controlled room with a 12-h light/dark cycle. Body weight was measured weekly. Body composition of the dams was measured at weaning and the offspring measured at 3 and 18 weeks of age using dual-energy X-ray absorptiometry (DXA) (Hologic QDR 4500; Hologic Inc., Marlborough, MA, USA). Each litter was considered as $n=1$ and male and female offspring were housed separately in plastic cages with wood-chip bedding following weaning at 3 weeks of age.

Distal jejunum and ileum tissue samples, and cecal digesta were collected at terminate points of dams (at weaning) and offspring (at 3 and 18 weeks of age) and immediately stored at $-80^{\circ} \mathrm{C}$ until use. Total bacterial DNA was isolated from cecal digesta using FastDNA spin kit for feces (MP Biomedicals, Lachine, QC, Canada), pre-treated with bead-beating (MP Biomedicals, Lachine, QC, Canada) for $40 \mathrm{~s} \times 3$ times. Purified DNA was quantified using Quant-iT ${ }^{\mathrm{TM}}$ PicoGreen $^{\mathrm{TM}}$ dsDNA Assay Kit (Invitrogen, Burlington, ON, Canada) and diluted to $20 \mathrm{ng} / \mu \mathrm{l}$ for $16 \mathrm{~S} \mathrm{rRNA}$ gene sequencing and $50 \mathrm{ng} / \mu \mathrm{l}$ for shotgun metagenomic sequencing.

\section{Analysis of Cecal Microbiota by $16 \mathrm{~S}$ rRNA Gene Sequencing}

To assess the contributions of maternal and offspring factors to cecal microbiota variations, genomic DNA from 105 samples were randomly selected for $16 \mathrm{~S}$ rRNA gene sequencing, corresponding to 19 dams at weaning, 36 offspring at 3 weeks of age, and 50 offspring at 18 weeks of age. Microbial 16S rRNA gene tags were sequenced on Illumina MiSeq platform $(2 \times 300 \mathrm{bp})$ by amplifying the hypervariable V3-V4 regions (Centre for Health Genomics and Informatics, Calgary, AB, Canada), and analyzed in QIIME2 platform (21) (QIIME2 2020.2). The quality controlled $16 \mathrm{~S}$ rRNA were filtered and denoised using DADA2 in QIIME2 (21) (QIIME2 2020.2). Frequency of sequence variants less than 10 were discarded. Taxonomy was assigned to sequence variants using Silva 138 database as reference. Sequence variants classified as genus Lactobacillus were additionally aligned to Genome Taxonomy Database release 95 (GTDB, https://gtdb.ecogenomic.org/) to reflect the current taxonomy of Lactobacillaceae (22) with the best BLAST hits. 


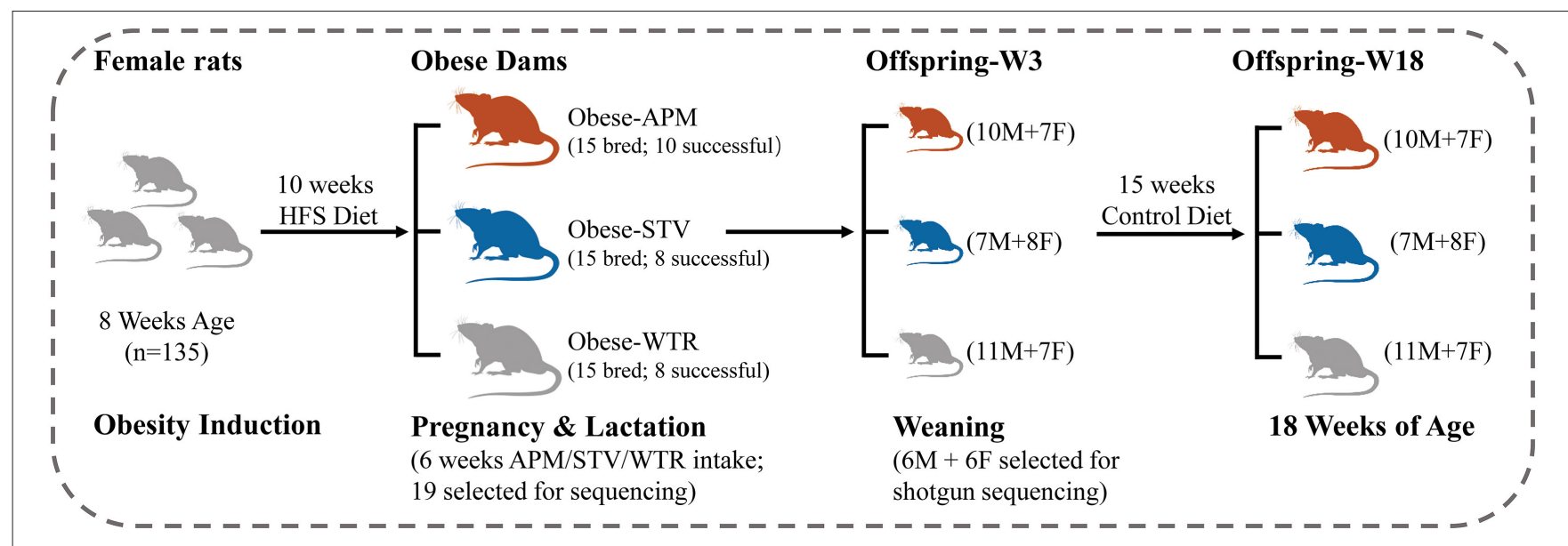

FIGURE 1 | Study design. HFS, high fat/sucrose diet; APM, aspartame; STV, stevia; WTR, water control; M, male; F, female; W, week.

\section{Recovery of Bacterial Genomes From Shotgun Metagenomic Reads}

To reconstruct cecal microbiome of offspring, 36 genomic DNAs from offspring at 3 weeks of age (weaning) were randomly selected for shotgun sequencing that included 12 samples (6 female and 6 males) from each dietary group. Shotgun metagenomic sequencing was conducted on NovaSeq $6000(2 \times$ $300 \mathrm{bp}$, Centre for Health Genomics and Informatics, Calgary, $\mathrm{AB}$, Canada) with NEB Ultra II library preparation protocol (New England Biolabs, Ipswich, MA, USA) to generate 400 $\mathrm{GB}$ of data $(800 \mathrm{M}$ reads). Due to the high percentage of the unidentified rodent microbiome and the high functional heterogeneity in microbial community at the strain level, we adopted de novo assembly-based metagenomic reconstruction to understand the complexity of functional alterations in offspring's gut microbiota $(23,24)$. Adapters were checked and removed by Trimmomatic (25). Quality-controlled reads were assembled into contigs/scaffolds by samples using default parameters in IDBA_UD (26). Long contigs (> 3,000 bp) were further clustered into bins using MaxBin2 (27) based on G $+\mathrm{C}$ content and abundance of contigs through ExpectationMaximization algorithm. The $16 \mathrm{~S}$ rRNA gene sequences data and shotgun metagenomic sequences data generated during this study are available at the National Center for Biotechnology Information (BioProject PRJNA675294) https://www.ncbi.nlm. nih.gov/biosample.

\section{Taxonomic Position and Relative Abundance of Metagenomic Assembled Genomes}

In total, 188 representative bins were chosen by de-replicating highly similar genomes using dRep (28), which refers to metagenomic assembled genomes (MAGs). Genomes completeness and contamination were estimated by examining a set of ubiquitous genes using CheckM (29) (Supplementary Table 1).
Phylogenetic trees of MAGs were built with PhyloPhlAn 3.0 (30) by aligning to a set of 400 core proteins. Species-, Genusand Family-level taxonomic labels were assigned to genomes when Mash (31) average genomic distance calculated from at least 100 makers was $<5 \%, 15 \%$ and $30 \%$ to the closest reference set, respectively (30). The phylogenetic tree was generated with Randomized Axelerated Maximum Likelihood (RAxML) method and visualized by iTOL (32). Raw reads were mapped back to MAGs to calculate relative abundance using CoverM with "relative_abundance" formula in "genome" model.

\section{Evaluation of Microbial Metabolism Potentials by Genome-Wide Screening}

To estimate the contribution of the cecal microbiome to microbial-derived energy harvest, metabolic enzymes were selected to retrieve pathways for microbial degradation of lactose and production of lactate, succinate, acetate, propionate, and butyrate (Supplementary Table 2). Query sequences for the same enzyme were selected from different bacteria and prealigned to minimize the overlap between BLAST hits for the same enzyme. Amino acid sequences of biochemically characterized key enzymes were blasted against amino acid database build from the open reading frames (ORF) of 188 assembled genomes (Prodigal 2.0) (33). An alignment coverage of $>70 \%, e$ value $\leq$ 1 e- 5 and amino acid identity of $\geq 40 \%$ or higher (34) were used as cut-off values for BLAST. The sums of mapped reads (\%) for all the positive hits for the same enzymes were calculated as their potential capacities to produce the corresponding metabolites in each sample.

\section{RNA Extraction and Quantification of Gene Expression}

Total RNA was extracted from jejunum and ileum tissue using Trizol (Invitrogen, Carlsbad, CA) and reversetranscribed into cDNA using SuperScriptII RT as previously described (35). Quantitative PCR was performed on BioRad iCycler (Bio-Rad, Hercules, USA) with primer sets LCT 
(forward, 5'-AATCTTCTTGGCTGGGAATGG-3'; reverse, $5^{\prime}$-CCTTGAGCACCTCGTTGA TG-3') and GATA4 (forward, 5'-AATCTTCTTGGCTGGGAATGG-3'; reverse, 5'-CCTTGA GCACCTCGTTGATG-3') targeted at lactase gene (lct) and regulatory gene (gata4). The $18 \mathrm{~S}$ gene was amplified as reference gene for relative quantification with primer set (forward, 5'-TGACTCAACACGGGAAACC-3'; reverse, 5'-TCGCTCCACCAACTAAGAAC- $3^{\prime}$ ). The relative expression ratio (R) of targets were calculated based on primer efficiency $(\mathrm{E})$ and the $\mathrm{Ct}$ deviations as follow: $\mathrm{R}=$

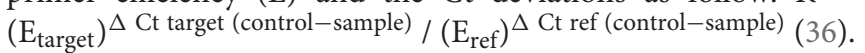

\section{Statistical Analysis}

The data for $16 \mathrm{~S}$ rRNA gene sequences and shotgun metagenomic sequences, including relative abundance of $16 \mathrm{~S}$ rRNA gene sequence variants, weighted UniFrac distances, relative abundance of assembled genomes and the corresponding prediction of metabolite production were compared using Kruskal-Wallis rank-sum test in $\mathrm{R}$ version 4.0.0 (2020-0424). Statistical significance of ANOSIM was determined through 999 permutations between dietary groups. The R value between 0 and 1 reflects the dissimilarity between the groups and was calculated as follows: $\mathrm{R}=$ difference of mean rank (all distances between groups - all distances within groups) / ( $\mathrm{N}(\mathrm{N}-1) / 4)$. The data for host parameters, including daily weight gain, body fat (\%), liver weight, and bone mineral density, and $\log _{10}$ transformed gene expression were analyzed using linear mixed-effects (LME) models in $\mathrm{R}$ version 4.0.0 0 (2020-04-24). Dietary treatment was treated as fixed factor; rat was considered as experimental unit and its random effect was removed. Correlations between genome, metabolite production and host parameters were analyzed by Spearman's rank method. Bonferroni-adjusted $p<0.05$ were considered significant.

\section{RESULTS}

\section{Maternal Consumption of Aspartame and Stevia Influences Gut Microbiota of Offspring}

Consumption of low-dose aspartame and stevia showed limited influence on the overall structure of cecal microbiota in dams (Figures 2A1,B1,C1; Supplementary Table 3) but significantly altered ( $p<0.001)$ cecal microbiota of their 3-week old offspring (Figures 2A2,B2,C2; Supplementary Table 3). The differences at 3 weeks gradually dissipated from weaning into adulthood (Figures 2A2,B3,C3; Supplementary Table 3). Dams and 18week-old offspring shared more similarities in cecal microbiota (Figure 2A1; Supplementary Table 3). Significant litter effects (male and female from same dam) were observed in 3-week old offspring, as indicated by the weighted UniFrac distances (Figure 2A2, R $=0.53, p=0.001$ ) and a higher number of highly similar ( $>99.0 \%$ average nucleotide identity) bacterial genomes that were shared between offspring from the same dam (Figure 2A3, $p<0.001$ ). Litter effects decreased in 18week-old offspring (Figure 2A2, $\mathrm{R}=0.07, p=0.0741$ ). No sex difference was observed in 3-week and 18-week $(p>0.05)$ old offspring. Therefore, subsequent metagenomic analyses focused on microbiome alterations in 3-week old offspring without stratification by sex.

\section{Metagenomic Reconstruction of Cecal Microbiome in 3-Week Old Rats}

In total, 188 genomic bins were recovered from 36 3-week old offspring cecal samples, with an average size of $3.23 \mathrm{Mb}$ and an average N50 of 74,759 bp (Supplementary Table 1). The high quality of reconstructed bacterial genomes enabled genome-based analysis of metabolic traits of intestinal microbial communities. Figure 3 depicts the phylogeny of 188 metagenomic assembled genomes. Five bacterial phyla were assigned to these 188 assembled genomes, including 1 assigned to Verrucomicrobia, 7 identified as Actinobacteria, 10 as Proteobacteria, 23 assigned to Bacteroidetes, and 137 identified as Firmicutes. Of the 188 assembled genomes, $149(79 \%)$ were assigned to genus or species level with an average amino acid identity of $>95 \%$ to the closest references calculated from at least 100 protein sequences. The relative abundance of each genome, referring to the percentage of mapped reads, was further transferred to Z-scores for visualization (Figure 4). In total, 92 genomes showed differential relative abundance $(p<0.05)$ between dietary treatments (Supplementary Figure 1).

\section{Reconstruction of Cecal SCFA Production Related Metabolic Pathways in 3-Week Old Rats}

To estimate the contribution of the cecal microbiome to microbial-derived energy harvest, pathways for microbial degradation of lactose and for production of lactate, succinate, acetate, propionate, and butyrate were reconstructed (Figure 4; Supplementary Table 1). Intracellular GH2 $\beta$-galactosidases BbgI, LacM, LacZ and Lacdeb, specifically distributed in Lachnospiraceae, Lactobacillaceae and Clostridiales were determined as the major metabolic enzymes hydrolysing milk-derived lactose in rats (Figures 4A1-A5). The relative abundance of these $\beta$-galactosidases significantly decreased in the stevia and/or aspartame groups compared to water control (Figures 4A1,A2,A4,A5, $p<0.05$ ). Phospho- $\beta$-galactosidase LacG (Figure 4A3) showed low abundance and no differences between sweeteners and water control groups in the cecal microbiota. The abundance of enzymes for pyruvate to lactate conversion (Figure 4B) was significantly reduced ( $p$ $<0.05)$ in the aspartame group compared to the stevia and water control.

Rat cecal microbes $(90.43 \%)$ were generally able to produce acetate through acetate kinase (AK) (not shown) and due to the multiple mapping of identical reads, the average of mapped read ratio for acetate was higher than $130 \%$. Variable bacterial types and abundance were involved in acetate production, but no difference was observed in total abundance between dietary groups (Figure 4A). Very few bacteria produce both propionate and butyrate as major products of carbohydrate fermentation (Supplementary Figure 1). Firmicutes genomes 


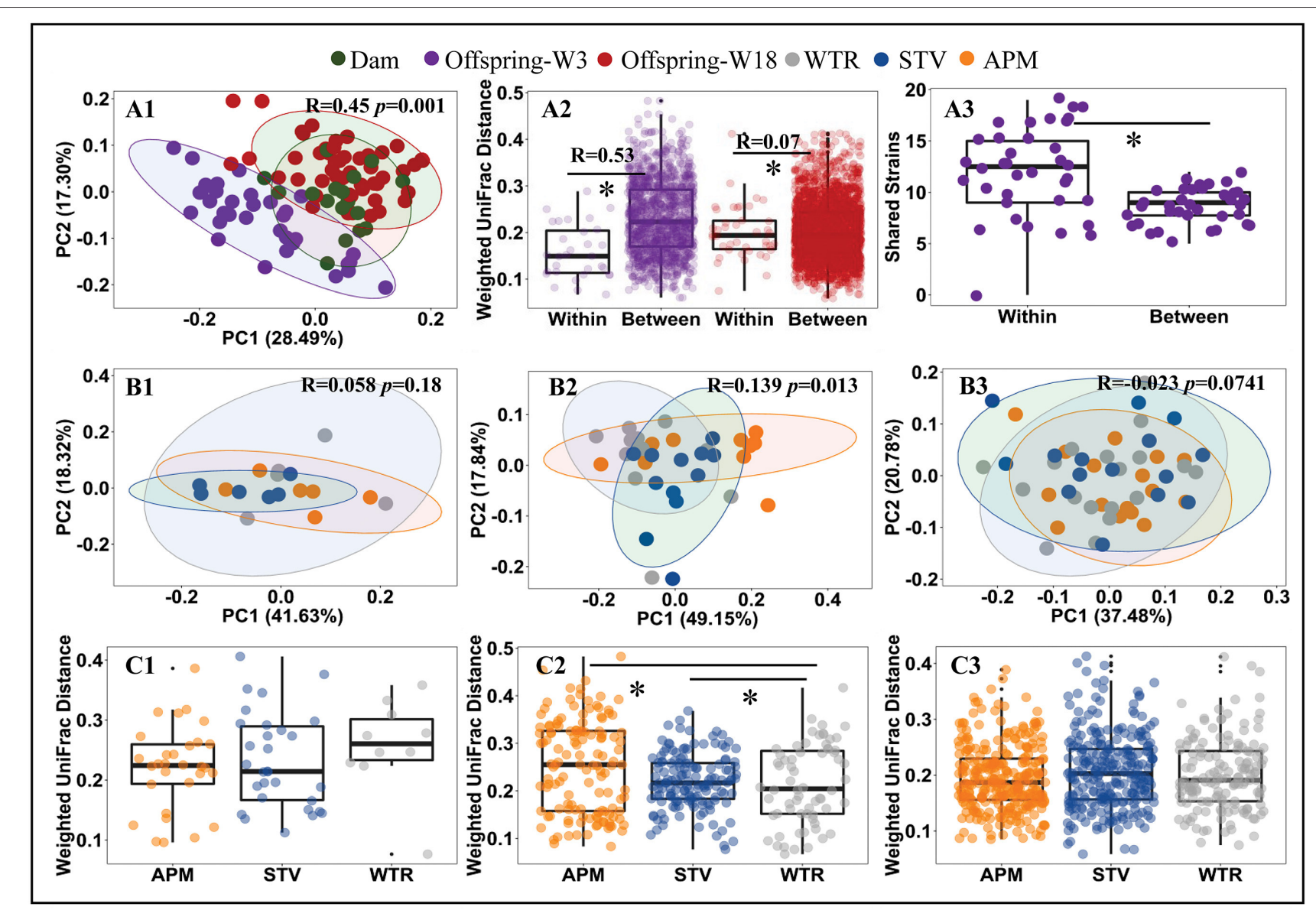

FIGURE 2 | Influence of maternal consumption of aspartame and stevia on cecal microbiota in rat dams and their offspring. Principle coordinate analysis (PCoA) (A1, B1-B3) of rat cecal microbiota; litter effects in offspring (A2); shared metagenomic assembled genomes (A3) between 3-week-old offspring from the same litter (left) and from different litters (right); Dietary effects in dams (C1), 3-week-old offspring (C2), and 18-week-old offspring (C3). Each dot represents an individual cecal sample. Data with asterisk $\left(^{*}\right)$ represents a significant difference $(p<0.05)$. W3, week 3; W18, week 18; APM, aspartame; STV, stevia; WTR, water control.

were identified as the major butyrate producers. Of these butyrate producers, 15 of 28 butyryl: acetate CoA-transferase carrying genomes and 10 of 17 butyrate kinase carrying genomes were significantly enriched in both aspartame and stevia groups or specifically enriched in aspartame $(p<$ 0.05). Of the three pathways for propionate production, the succinate pathway, dominated by Akkermansia muciniphila and 14_Bacteroidaceae unclassified, was the most abundant (30 of 46 propionate-producing genomes). This pathway was significantly enriched in the aspartame group. Of the 30 succinate-propionate producers, 9 genomes were enriched by aspartame and stevia or only by aspartame (4 of 9). The propanediol pathway for propionate production was less abundant in the stevia group although the production of 1,2propanediol was not different between dietary groups. Genes coding for the acrylate pathway were rare in the rat cecal microbiome. Production of cobalamin (vitamin B12), a co-factor required by multiple metabolic processes including propionate production, also showed variable abundances in aspartame and/or stevia offspring.

\section{Reducing Dimensions of Microbial Population Differences and Their Correlations With Host Growth and Body Composition}

To further explore the influence of non-nutritive sweeteners on the cecal microbiome of offspring, genomes with high relative abundance and significant differences between groups were selected as representatives for further correlation analysis (Figure 5A; Supplementary Table 4). The genome of 14_Bacteroidaceae unclassified represented the most abundant microbe that was enriched in both aspartame and stevia groups; the genome of 1_Akkermansia muciniphila represented bacteria specifically enriched by aspartame; microbes reduced by aspartame were represented by 48_Limosilactobacillus reuteri and 49_Ligilactobacillus murinus. The other genomes, including 176_Eubacterium spp., 151_Blautia spp., 152_Blautia spp., and 175_Lachnospiraceae unclassified, showed variable differences between dietary treatments. Jejunal and ileal expression of lactase and its regulator GATA4 can directly affect the availability of 


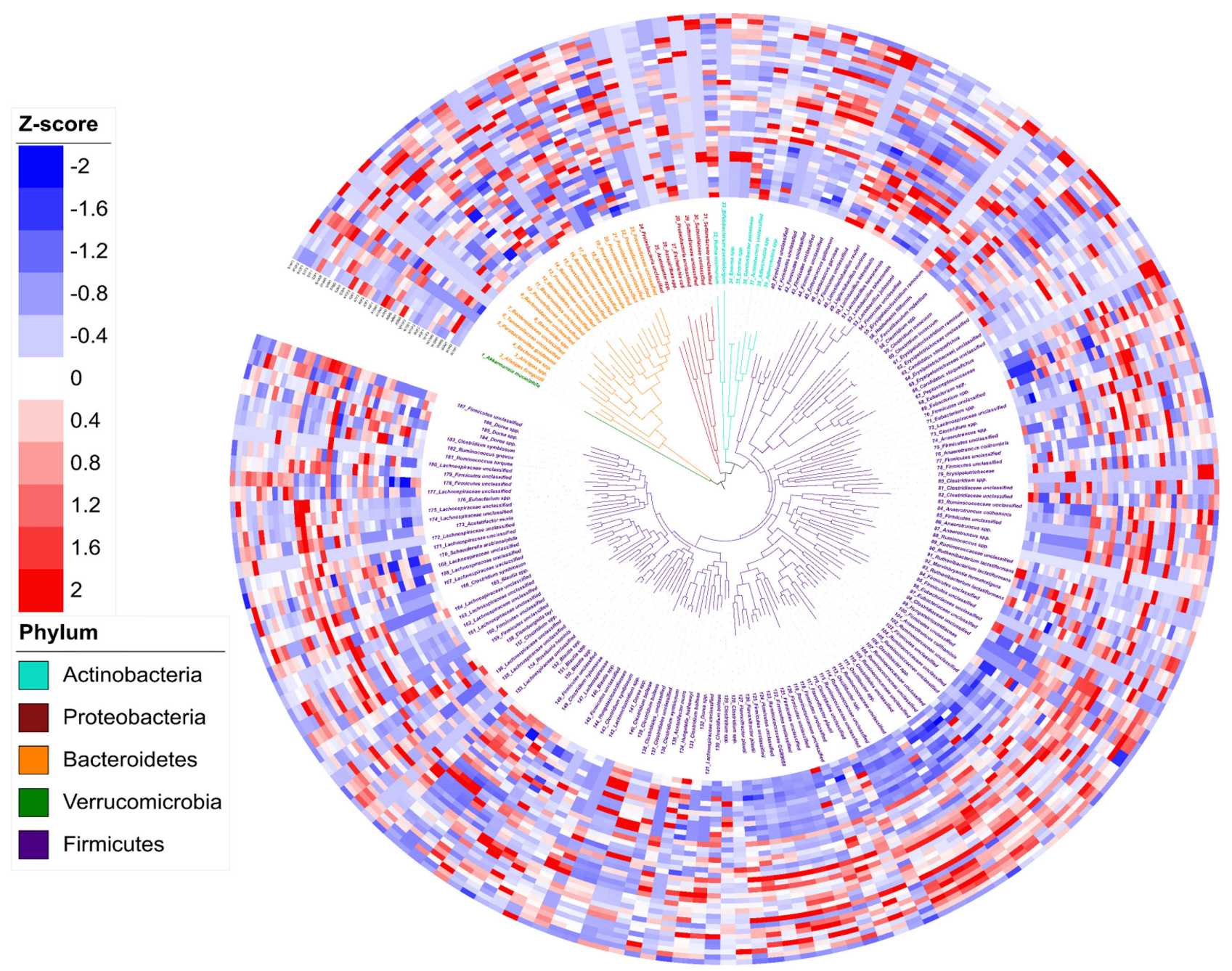

FIGURE 3 | Phylogeny and abundance of metagenomic assembled genomes recovered from 3-week old rat cecal digesta. The relative abundance of bins was calculated from percentage of re-mapped reads in the sample and presented as Z-score: Z-score = (relative abundance - mean of relative abundance)/standard deviation. Phylogenetic tree (the innermost layer) and taxonomic affiliations (the middle layer) of 188 metagenomic assembled genomes (MAGs). Tree branches and labels with different colors represent different phyla as indicated by the color code in the lower left. The outermost heatmap depicts the relative abundance of the 188 bins in each sample, inside to outside: Water Control (12 layers), Aspartame (12 layers) and Stevia (12 layers).

lactose for fermentation in the cecum. The expression of these genes was therefore examined as a potential upstream factor shaping the microbiome, however no significant differences between dietary treatments were found (Figure 5B). Host parameters, including daily weight gain, body fat, liver weight, and bone mineral density were selected for correlation analysis (Figure 5C). Maternal consumption of aspartame increased daily weight gain in offspring (Figure 5C). Aspartame and stevia significantly increased body fat $\%$ and liver weight but decreased bone mineral density in 3-week old offspring (Figure 5C).

Correlations between bacterial genomes, bacterial metabolites, and between host parameters depict microbialhost interactions in weanling pups (Figure 5D). Two distinct bacterial consortia were identified, showing negative correlations with each other and consistently opposite correlations with metabolite production and host parameters. The propionate- and butyrate-producing consortium, represented by 1_Akkermansia muciniphila, 14_Bacteroidaceae unclassified, 175_Lachnospiraceae unclassified, and 176_Eubacterium spp., was enriched in offspring of sweetener-consuming dams, and was positively correlated with offspring's daily body weight gain, liver weight and body fat but negatively correlated with bone mineral density (Figure 5D). Conversely, the lactateproducing consortium, including 48_Limosilactobacillus reuteri, 49_Ligilactobacillus murinus, 151_Blautia spp., and 152_Blautia $s p p$., which degrade lactose into lactate, were negatively correlated to the host parameters mentioned above except bone mineral density (Figure 5D). Significant correlations between gene expression of lactase/GATA4 gene and selected bacterial 


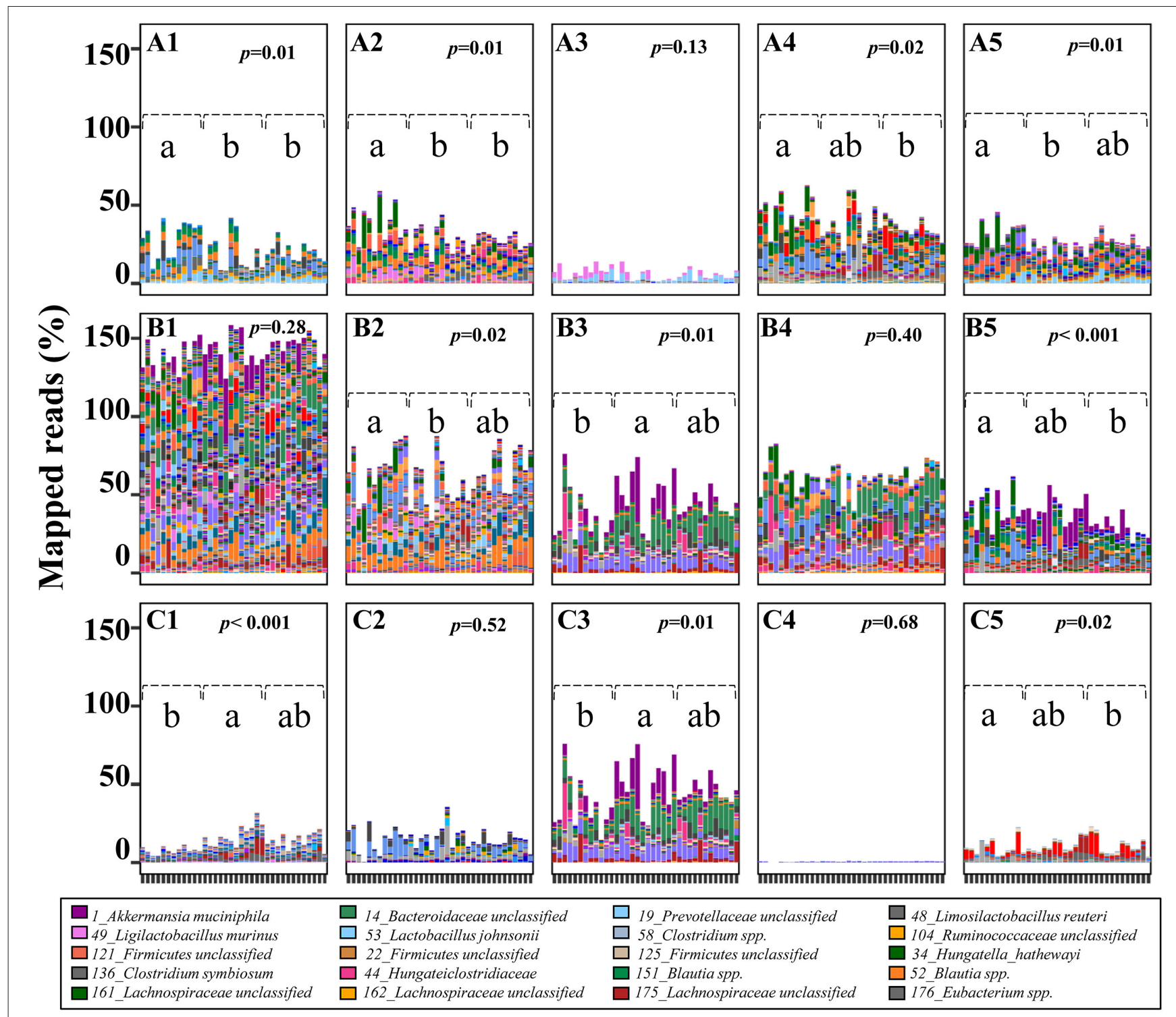

FIGURE 4 | Predicted potential of cecal microbiome involved in lactose and SCFA metabolism in 3-week old offspring. The potential of each cecal microbial community to degrade lactose and generate SCFA-related metabolites was calculated by the sum of mapped reads (\%) for positive hits of the corresponding key enzymes as follow: Lactose (A1,A2) beta-galactosidase (LacZ and Lacdeb), (A3-A5) phospho- $\beta$-galactosidase (LacG, Bbgl and LacM); Acetate (B1) acetate kinase (AK); Lactate (B2) D/L-lactate dehydrogenase (LacDeh); Succinate, (B3) fumarate reductase (frdA); 1,2-Propanediol; (B4), lactaldehyde reductase (LacRd); Cobalamin (Vitamin B12), (B5) cobalt transporter protein (CbiM); Butyrate (C1) butyryl:acetate CoA-transferase (BCoA), (C2) butyrate kinase (BK); Propionate, (C3) methylmalonyl-CoA decarboxylase (McoA), (C4) lactoyl-CoA dehydratase (LacCoRd), (C5) propanediol dehydratase (pduCDE). Data with different superscripts represents significant difference $(p<0.05)$. For bars in each panel, left to right: water control $(n=6)$; aspartame $(n=6)$ and stevia $(n=6)$.

genomes and host parameters were only observed between jejunal lct/gata4 with butyrate formation (Figure 5D).

\section{DISCUSSION}

In this study, we demonstrate that the altered intestinal microbiota resulting from consumption of non-nutritive sweeteners by dams explains the negative metabolic changes occurring in their offspring that were themselves never directly exposed to the sweeteners. To identify possible mechanisms by which this intergenerational risk is transmitted, we assessed the metagenomic reconstruction of intestinal metabolism of dietary carbohydrates in the offspring alongside host parameters including weight gain, body fat, liver weight and bone mineral density. We show that maternal consumption of aspartame or stevia altered microbial metabolism in the offspring; specifically, the relative abundance of propionate- and butyrate-producing species increased, and the abundance of lactose-fermenting species decreased in offspring at weaning. The resultant altered propionate and lactate production could explain increased body 


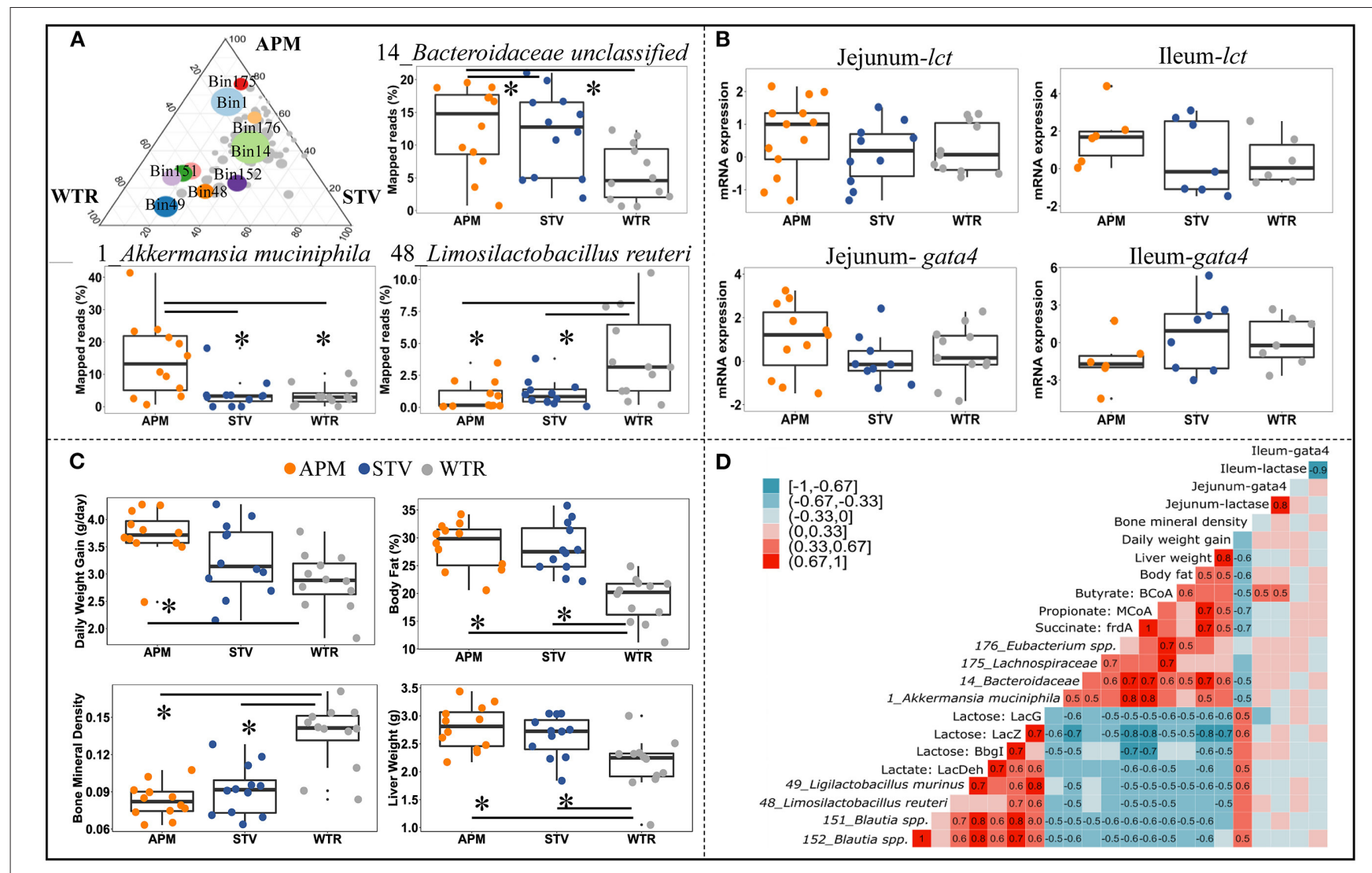

FIGURE 5 | Connections between cecal microbiome alterations and host parameters in 3-week old offspring. The top 10 influenced genomes in cecal microbiota of 3-week-old offspring (ternary plot) and the relative abundance of 3 representative influenced genomes (boxplots) (A); mRNA expression fold change of jejunal/ileal lactase gene (Ict) and GATA4 (gata4) (B); Growth peformance and body composition of weanlings (C); The coefficient matrix of Spearman's rank correlations between the most influenced genomes, metabolic enzymes, gene expression, and the corresponding growth and body composition of 3-week old offspring (D). Data with asterisk $\left(^{*}\right)$ represents significant difference $(p<0.05)$. APM, aspartame; STV, stevia; WTR, water control.

weight and body fat in offspring from aspartame and stevia consuming dams. Importantly, we show that sweet tastants have a lasting and intergenerational effect on gut microbiota, microbial metabolites and host health.

Artificial sweeteners induce compositional and functional alterations in gut microbiota of human and rats $(5,8,9)$. These shifts in gut microbiota have been linked to the development of obesity-related glucose intolerance which is transferable to germ-free mice through fecal microbiota transplantation $(5,9$, 37). Maternal dietary intake during gestation and lactation is known to program offspring health via multiple mechanisms (37-39), which include transmission of gut microbiota and its metabolic potential $(37,40)$. We examined litter effects on microbiota structural similarities and shared identical bacterial genomes between offspring from the same dam vs. different dams and confirmed that maternal microbial transmission plays an important role in the development of cecal microbiota of 3-week old offspring.

Given the obese phenotype of offspring derived from dams consuming non-nutritive sweeteners, we focused our metagenomics reconstruction on the pathways for the production of acetate, propionate, and butyrate. We observed increased readouts of succinate/propionate conversion and butyrate formation in the cecal microbiome of offspring from aspartame and stevia consuming dams and their positive correlation with increased liver weight, body weight gain, and body fat. Altered SCFA metabolism, especially elevated serum and fecal propionate, has been shown previously in obese animals following artificial sweetener consumption $(3,5,8,9)$. Exogenous administration of propionate to healthy individuals in a meal resulted in hyperglycemia which was primarily attributed to an increase in serum glucagon and overexpression of fatty acid-binding protein4 (5). Beyond higher blood glucose and hyperinsulinemia, significantly higher body weight gain and body fat was also observed in mice that were administered a chronic low dose of propionate in their drinking water compared to control mice (41). These findings are in line with the impaired glucose tolerance we observed in male offspring of our aspartame dams (9). We also observed increased mRNA levels of dopamine transporter in the ventral tegmental area of the male weanlings of 
sweetener consuming dams (9). Upregulation of the dopamine transporter could imply an activation of the mesolimbic reward pathway in the brain that promotes food-seeking behavior, particularly to highly palatable foods (9). In depth analysis of central and systemic appetite regulation in offspring of sweetener consuming dams, including the satiety hormones, PYY and GLP-1, are warranted to better understand the influence of altered microbial metabolism on appetite regulation in these animals $(41,42)$.

Understanding how shifts in endogenous propionate production affect the development of metabolic abnormalities, however, remains challenging. We show that maternal intake of aspartame and stevia and the resultant early colonizers that are transmitted from dams impacted the cecal microbiome of offspring. Host diet and particularly the content of indigestible but fermentable carbohydrate plays a key role in shaping the gut microbiome (43). Milk-derived lactose accounts for $12-15 \%$ of rat milk solids during late lactation $(44,45)$. In human infants and suckling rats, a substantial proportion of dietary lactose escapes small intestinal hydrolysis and absorption and is fermented in the large intestine $(46,47)$. The cecal/colonic lactate that is produced from lactose fermentation in turn serves as a substrate for butyrate and propionate production by other members of the intestinal microbiota (48-50). Therefore, we extended our pathway construction to lactose/lactate metabolism and identified the reduced proportions of metagenomic reads mapped to lactose hydrolase and lactate formation, and their negative correlations with propionate/butyrate production and weight gain and body fat in offspring from dams that consumed nonnutritive sweeteners. Notably, host glycans, mainly mucin, are degraded predominantly by Akkermansia and Bacteroides species $(51,52)$. In line with the highly enriched glycan degradation pathways found in mice consuming saccharin (5), the increased relative abundance of Akkermansia and Bacteroidetes with a concomitant lower proportion of lactobacilli in our study also suggests that maternal consumption of non-nutritive sweeteners increases the relevance of host glycans but decreases the relevance of lactose as substrate for cecal carbohydrate fermentation in their offspring. Decreased lactate formation from lactose by lactobacilli results in an increased cecal $\mathrm{pH}$, which further benefits the mucin-degrading Akkermansia and Bacteroidetes $(5,53,54)$ and shifts cecal microbial metabolism $(34,50)$.

More research is needed to evaluate the possible role of nonnutritive sweeteners on offspring metabolism via their presence in breastmilk. Important to the current investigation is that while some low-calorie sweeteners have been detected in breastmilk (i.e., saccharin, sucralose, and acesulfame-potassium), this does not appear to be true for all sweeteners including aspartame (6). In addition, the concentration of non-nutritive sweeteners in breast milk after maternal consumption, even if detectable, is too low to impact gut microbiota through direct provision of any substrate and these sweeteners are not known to alter lactose content of milk or host glycans secretions $(2,6,15,55)$. Given the impact of jejunal and ileal lactase activity on the availability of lactose for intestinal microbiota in suckling rats and the decline in lactase activity which is essentially regulated at the posttranscriptional level during the late lactation period (56-59), we examined the gene expression of jejunal/ileal lactase and regulator GATA4 but did not find a difference between offspring of dams consuming non-nutritive sweeteners or not. Although we cannot rule out other possibilities, the lack of difference reduces the likelihood that lactose metabolism had an upstream effect on cecal SCFA production and suggests that obesity risk in offspring after maternal consumption of non-nutritive sweeteners is mediated by the host response to an altered pattern of bacterial metabolites. The possibility remains that exposure to altered bacterial metabolites that have previously been detected in the maternal fecal and/or serum metabolome when mothers consume sucralose and aspartame $(3,60)$, could contribute.

\section{CONCLUSIONS}

Our metagenomic reconstruction of cecal SCFAs metabolism identified decreased lactose fermentation and an altered propionate and lactate production as the central tenet of increased body weight and body fat in offspring from aspartame and stevia consuming dams. Although the mechanisms of transmission of this phenotype from mother to offspring and its perpetuation into adulthood remain to be elucidated, this study demonstrates the intergenerational effect that sweet tastants have on gut microbiota, microbial metabolites and host health. This result has important implications for human health because the diet of mothers during pregnancy and lactation likely also impacts the gut microbiota, microbial metabolites, and the metabolic fitness to their children. Compositional and functional shifts in the microbiome with non-nutritive sweetener consumption should be investigated further in human cohorts to inform guidelines for maternal diet during pregnancy.

\section{DATA AVAILABILITY STATEMENT}

The datasets presented in this study can be found in online repositories. The names of the repository/repositories and accession number(s) can be found below: https://www.ncbi.nlm. nih.gov/, BioProject PRJNA675294.

\section{ETHICS STATEMENT}

Ethical approval was granted by the University of Calgary Animal Care Committee (Protocol\#AC15-0079).

\section{AUTHOR CONTRIBUTIONS}

WW designed research, prepared samples, performed bioinformatics analysis, generated figures, and wrote paper. $\mathrm{JN}$ designed and performed original animal experiments and reviewed the paper. MG assisted with analysis and interpretation and reviewed the paper. RR designed research, assisted with interpretation, obtained funding, and had primary responsibility 
for final content. All authors have read and approved the final manuscript.

\section{FUNDING}

This work was supported by the Natural Sciences and Engineering Research Council of Canada through grant RGPIN2016-03773. WW is supported by a University of Calgary Eyes High Postdoctoral Fellowship. JN was supported by Alberta Children's Hospital Research Institute and Canadian Institutes of Health Research. MG is supported by the Canada Research Chairs Program.

\section{REFERENCES}

1. Rogers PJ. The role of low-calorie sweeteners in the prevention and management of overweight and obesity: evidence v. conjecture. Proc Nutr Soc. (2018) 77:230-8. doi: 10.1017/S0029665117004049

2. Olivier-Van Stichelen S, Rother KI, Hanover JA. Maternal exposure to nonnutritive sweeteners impacts progeny's metabolism and microbiome. Front Microbiol. (2019) 10:1360. doi: 10.3389/fmicb.2019.01360

3. Palmnäs MSA, Cowan TE, Bomhof MR, Su J, Reimer RA, Vogel HJ, et al. Low-dose aspartame consumption differentially affects gut microbiotahost metabolic interactions in the diet-induced obese rat. PLoS ONE. (2014) 9:e109841. doi: 10.1371/journal.pone.0109841

4. Magnuson BA, Burdock GA, Doull J, Kroes RM, Marsh GM, Pariza MW, et al. Aspartame: A safety evaluation based on current use levels, regulations, and toxicological and epidemiological studies. Crit Rev Toxicol. (2007) 37:629727. doi: 10.1080/10408440701516184

5. Suez J, Korem T, Zeevi D, Zilberman-Schapira G, Thaiss CA, Maza O, et al. Artificial sweeteners induce glucose intolerance by altering the gut microbiota. Nature. (2014) 514:181-6. doi: 10.1038/nature13793

6. Sylvetsky AC, Gardner AL, Bauman V, Blau JE, Garraffo HM, Walter PJ, et al. Nonnutritive sweeteners in breast milk. J Toxicol Environ Heal Part A. (2015) 78:1029-32. doi: 10.1080/15287394.2015.1053646

7. Azad MB, Sharma AK, De Souza RJ, Dolinsky VW, Becker AB, Mandhane PJ, et al. Association between artificially sweetened beverage consumption during pregnancy and infant body mass index. JAMA Pediatr. (2016) 170:66270. doi: 10.1001/jamapediatrics.2016.0301

8. Kuk JL, Brown RE. Aspartame intake is associated with greater glucose intolerance in individuals with obesity. Appl Physiol Nutr Metab. (2016) 41:795-8. doi: 10.1139/apnm-2015-0675

9. Nettleton JE, Cho NA, Klancic T, Nicolucci AC, Shearer J, Borgland SL, et al. Maternal low-dose aspartame and stevia consumption with an obesogenic diet alters metabolism, gut microbiota and mesolimbic reward system in rat dams and their offspring. Gut. (2020) 69:1807-17. doi: 10.1136/gutjnl-2018-31 7505

10. Roberts A, Renwick AG. Comparative toxicokinetics and metabolism of rebaudioside A, stevioside, and steviol in rats. Food Chem Toxicol. (2008) 46:S31-9. doi: 10.1016/j.fct.2008.05.006

11. Renwick AG, Tarka SM. Microbial hydrolysis of steviol glycosides. Food Chem Toxicol. (2008) 46:S70-4. doi: 10.1016/j.fct.2008.05.008

12. Gardana C, Simonetti P, Canzi E, Zanchi R, Pietta P. Metabolism of stevioside and rebaudioside A from stevia rebaudiana extracts by human microflora. $J$ Agric Food Chem. (2003) 51:6618-22. doi: 10.1021/jf0303619

13. Lipton WE Li YN, Younoszai MK, Stegink LD. Intestinal absorption of aspartame decomposition products in adult rats. Metabolism. (1991) 40:133745. doi: 10.1016/0026-0495(91)90040-4

14. Ranney RE, Oppermann JA, Muldoon E, McMahon FG. Comparative metabolism of aspartame in experimental animals and humans. J Toxicol Environ Health. (1976) 2:441-51. doi: 10.1080/15287397609529445

15. Koyama E, Sakai N, Ohori Y, Kitazawa K, Izawa O, Kakegawa K, et al. Absorption and metabolism of glycosidic sweeteners of stevia mixture and

\section{ACKNOWLEDGMENTS}

The authors would like to acknowledge Shelly Wegener and Dr. Richard Pon, Centre for Health Genomics and Informatics at the University of Calgary for support with the 16S rRNA and shotgun sequencing.

\section{SUPPLEMENTARY MATERIAL}

The Supplementary Material for this article can be found online at: https://www.frontiersin.org/articles/10.3389/fnut.2021. 795848/full\#supplementary-material

their aglycone, steviol, in rats and humans. Food Chem Toxicol. (2003) 41:875-83. doi: 10.1016/S0278-6915(03)00039-5

16. Canfora EE, Jocken JW, Blaak EE. Short-chain fatty acids in control of body weight and insulin sensitivity. Nat Publ Gr. (2015) 11:57791. doi: 10.1038/nrendo.2015.128

17. Chleilat F, Schick A, Deleemans JM, Ma K, Alukic E, Wong J, et al. Paternal high protein diet modulates body composition, insulin sensitivity, epigenetics, and gut microbiota intergenerationally in rats. FASEB J. (2021) 35:e21847. doi: 10.1096/FJ.202100198RR

18. Levin BE, Dunn-Meynell AA, Balkan B, Keesey RE. Selective breeding for dietinduced obesity and resistance in Sprague- Dawley rats. Am J Physiol Regul Integr Comp Physiol. (1997) 273:725-30. doi: 10.1152/ajpregu.1997.273.2.r725

19. Nutrition Guideline Sugar Substitutes. Alberta Heal Serv. (2020). Available online at: https://www.albertahealthservices.ca/assets/info/nutrition/if-nfsng-sugar-substitutes.pdf

20. List of Permitted Sweeteners (Lists of Permitted Food Additives) Canada.ca (2021). Available online at: https://www.canada.ca/en/healthcanada/services/food-nutrition/food-safety/food-additives/lists-permitted/ 9-sweeteners.html.

21. Bolyen E, Rideout JR, Dillon MR, Bokulich NA, Abnet CC, AlGhalith GA, et al. Reproducible, interactive, scalable and extensible microbiome data science using QIIME 2. Nat Biotechnol. (2019) 37:8527. doi: 10.1038/s41587-019-0209-9

22. Zheng J, Wittouck S, Salvetti E, Franz CMAP, Harris HMB, Mattarelli P, et al. A taxonomic note on the genus Lactobacillus: description of 23 novel genera, emended description of the genus Lactobacillus beijerinck 1901, and union of Lactobacillaceae and Leuconostocaceae. Int J Syst Evol Microbiol. (2020) 70:2782-858. doi: 10.1099/ijsem.0.004107

23. Segata N. On the road to strain-resolved comparative metagenomics. mSystems. (2018) 3:e00190-17. doi: 10.1128/MSYSTEMS.00190-17

24. Quince C, Walker AW, Simpson JT, Loman NJ, Segata N. Shotgun metagenomics, from sampling to analysis. Nat Biotechnol. (2017) 35:83344. doi: $10.1038 /$ nbt.3935

25. Bolger AM, Lohse $M$, Usadel B. Trimmomatic: A flexible trimmer for Illumina sequence data. Bioinformatics. (2014) 30:2114-20. doi: 10.1093/bioinformatics/btu170

26. Peng Y, Leung HCMM, Yiu SM, Chin FYLL, IDBA-UD. A de novo assembler for single-cell and metagenomic sequencing data with highly uneven depth. Bioinformatics. (2012) 28:1420-8. doi: 10.1093/bioinformatics/bts174

27. Wu YW, Simmons BA. Singer SW. MaxBin 20: An automated binning algorithm to recover genomes from multiple metagenomic datasets. Bioinformatics. (2016) 32:605-7. doi: 10.1093/bioinformatics/btv638

28. Olm MR, Brown CT, Brooks B, Banfield JF. DRep: A tool for fast and accurate genomic comparisons that enables improved genome recovery from metagenomes through de-replication. ISME J. (2017) 11:28648. doi: 10.1038/ismej.2017.126

29. Parks DH, Imelfort M, Skennerton CT, Hugenholtz P, Tyson GW. CheckM: Assessing the quality of microbial genomes recovered from isolates, single cells, and metagenomes. Genome Res. (2015) 25:104355. doi: 10.1101/gr.186072.114 
30. Asnicar F, Thomas AM, Beghini F, Mengoni C, Manara S, Manghi P. et al. Precise phylogenetic analysis of microbial isolates and genomes from metagenomes using PhyloPhlAn 30. Nat Commun. (2020) 11:110. doi: 10.1038/s41467-020-16366-7

31. Ondov BD, Treangen TJ, Melsted P, Mallonee AB, Bergman NH, Koren S, et al. Mash: Fast genome and metagenome distance estimation using MinHash. Genome Biol. (2016) 17:1-14. doi: 10.1186/s13059-016-0997-x

32. Stamatakis A. RAxML version 8: A tool for phylogenetic analysis and post-analysis of large phylogenies. Bioinformatics. (2014) 30:13123. doi: 10.1093/bioinformatics/btu033

33. Vent DH, Indian S, Zhao S, Zheng P, Dong S, Zhan X, et al. Prodigal: prokaryotic gene recognition and translation initiation site identification. Nat Commun. (2014) 6:1-8. doi: 10.3389/fgene.2015.00348

34. Reichardt N, Duncan SH, Young P, Belenguer A, McWilliam Leitch C, Scott KP, et al. Phylogenetic distribution of three pathways for propionate production within the human gut microbiota. ISME J. (2014) 8:132335. doi: 10.1038/ismej.2014.14

35. Reid DT, Eller LK, Nettleton JE, Reimer RA. Postnatal prebiotic fibre intake mitigates some detrimental metabolic outcomes of early overnutrition in rats. Eur J Nutr. (2016) 55:2399-409. doi: 10.1007/s00394-015-1047-2

36. Pfaffl MW, A. new mathematical model for relative quantification in real-time RT-PCR. Nucleic Acids Res. (2001) 29:2002-7. doi: 10.1093/nar/29.9.e45

37. Kimura I, Miyamoto J, Ohue-Kitano R, Watanabe K, Yamada T, Onuki M, et al. Maternal gut microbiota in pregnancy influences offspring metabolic phenotype in mice. Science. (2020) 367:8429. doi: 10.1126/science.aaw8429

38. Jašarević E, Bale TL. Prenatal and postnatal contributions of the maternal microbiome on offspring programming. Front Neuroendocrinol. (2019) 55:100797. doi: 10.1016/j.yfrne.2019.100797

39. Hsu CN, Tain YL. The good, the bad, and the ugly of pregnancy nutrients and developmental programming of adult disease. Nutrients. (2019) 11:894. doi: 10.3390/nu11040894

40. Stiemsma LT, Michels KB. The tole of the microbiome in the developmental origins of health and disease. Pediatrics. (2018) 141:e20172437. doi: 10.1542/peds.2017-2437

41. Tirosh A, Calay ES, Tuncman G, Claiborn KC, Inouye KE, Eguchi K, et al. The short-chain fatty acid propionate increases glucagon and FABP4 production, impairing insulin action in mice and humans. Sci Transl Med. (2019) 11:120. doi: 10.1126/scitranslmed.aav0120

42. Flint HJ, Scott KP, Louis P, Duncan SH, Flint HJ, Scott KP, et al. The role of the gut microbiota in nutrition and health. Nat Rev Gastroenterol Hepatol. (2012) 9:577-89. doi: 10.1038/nrgastro.2012.156

43. Carmody RN, Gerber GK, Luevano JM, Gatti DM, Somes L, Svenson KL, et al. Diet dominates host genotype in shaping the murine gut microbiota. Cell Host Microbe. (2015) 17:72-84. doi: 10.1016/j.chom.2014.11.010

44. Najjar AM, Parsons PM, Duncan AM, Robinson LE, Yada RY, Graham TE. The acute impact of ingestion of breads of varying composition on blood glucose, insulin and incretins following first and second meals. Br J Nutr. (2009) 101:391-8. doi: 10.1017/S0007114508003085

45. Kuhn NJ. The lactose and neuraminlactose content of rat milk and mammary tissue. Biochem J. (1972) 130:177-80. doi: 10.1042/bj1300177

46. Kim K, Benevenga NJ, Grummer RH. Estimation of the fraction of the lactose in a high lactose diet available for fermentation in the cecum and colon of the rat. J Nutr. (1978) 108:79-89. doi: 10.1093/jn/108.1.79

47. Francavilla R, Calasso M, Calace L, Siragusa S, Ndagijimana M, Vernocchi $\mathrm{P}$, et al. Effect of lactose on gut microbiota and metabolome of infants with cow's milk allergy. Pediatr Allergy Immunol. (2012) 23:4207. doi: 10.1111/j.1399-3038.2012.01286.x

48. Louis P, Flint HJ. Formation of propionate and butyrate by the human colonic microbiota. Environ Microbiol. (2017) 19:29-41. doi: 10.1111/1462-2920.13589

49. Duncan SH, Louis P, Flint HJ. Lactate-utilizing bacteria, isolated from human feces, that produce butyrate as a major fermentation product. Appl Environ Microbiol. (2004) 70:5810-7. doi: 10.1128/AEM.70.10.5810-5817.2004
50. Muñoz-Tamayo R, Laroche B, Walter É, Doré J, Duncan SH, Flint HJ, et al. Kinetic modelling of lactate utilization and butyrate production by key human colonic bacterial species. FEMS Microbiol Ecol. (2011) 76:61524. doi: 10.1111/j.1574-6941.2011.01085.x

51. Ottman N, Davids M, Suarez-Diez M, Boeren S, Schaap PJ, dos Santos VAPM, et al. Genomescale model and omics analysis of metabolic capacities of Akkermansia muciniphila reveal a preferential mucin-degrading lifestyle. Appl Environ Microbiol. (2017) 83:e01014-17. doi: 10.1128/AEM.01 014-17

52. Tailford LE, Crost EH, Kavanaugh D, Juge N. Mucin glycan foraging in the human gut microbiome. Front Genet. (2015) 5:00081. doi: 10.3389/fgene.2015.00081

53. Ping Wang S, Rubio LA, Duncan SH, Donachie GE, Holtrop G, Lo G, et al. Pivotal roles for $\mathrm{pH}$, lactate, and lactate-utilizing bacteria in the stability of a human colonic microbial ecosystem. Mol Biol Physiol. (2020) 5:64565. doi: 10.1128/mSystems.00645-20

54. Jiang $\mathrm{T}$, Savaiano DA. Modification of colonic fermentation by bifidobacteria and $\mathrm{pH}$ in vitro: Impact on lactose metabolism, short-chain fatty acid, and lactate production. Dig Dis Sci. (1997) 42:2370-7. doi: 10.1023/A:1018895524114

55. Araújo JR, Martel F, Keating E. Exposure to non-nutritive sweeteners during pregnancy and lactation: impact in programming of metabolic diseases in the progeny later in life. Reprod Toxicol. (2014) 49:196201. doi: 10.1016/j.reprotox.2014.09.007

56. Rings EHHM, Krasinski SD, Van Beers EH, Moorman AFM, Dekker J, Montgomery RK, et al. Restriction of lactase gene expression along the proximal-to-distal axis of rat small intestine occurs during postnatal development. Gastroenterology. (1994) 106:1223-32. doi: 10.1016/0016-5085(94)90013-2

57. Freund JN, Duluc I, Raul F. Lactase expression is controlled differently in the jejunum and ileum during development in rats. Gastroenterology. (1991) 100:388-94. doi: 10.1016/0016-5085(91)90207-2

58. van Wering HM, Bosse T, Musters A, de Jong E, de Jong N, Hogen Esch CE, et al. Complex regulation of the lactase-phlorizin hydrolase promoter by GATA-4. Am J Physiol Liver Physiol. (2004) 287:G899909. doi: 10.1152/ajpgi.00150.2004

59. Motohashi Y, Fukushima A, Kondo T, Sakuma K. Lactase decline in weaning rats is regulated at the transcriptional level and not caused by termination of milk ingestion. J Nutr. (1997) 127:1737-43. doi: 10.1093/jn/127. 9.1737

60. Bian X, Chi L, Gao B, Tu P, Ru H, Lu K. Gut microbiome response to sucralose and its potential role in inducing liver inflammation in mice. Front Physiol. (2017) 8:487. doi: 10.3389/FPHYS.2017.00487/ BIBTEX

Conflict of Interest: The authors declare that the research was conducted in the absence of any commercial or financial relationships that could be construed as a potential conflict of interest.

Publisher's Note: All claims expressed in this article are solely those of the authors and do not necessarily represent those of their affiliated organizations, or those of the publisher, the editors and the reviewers. Any product that may be evaluated in this article, or claim that may be made by its manufacturer, is not guaranteed or endorsed by the publisher.

Copyright (c) 2022 Wang, Nettleton, Gänzle and Reimer. This is an open-access article distributed under the terms of the Creative Commons Attribution License (CC $B Y)$. The use, distribution or reproduction in other forums is permitted, provided the original author(s) and the copyright owner(s) are credited and that the original publication in this journal is cited, in accordance with accepted academic practice. No use, distribution or reproduction is permitted which does not comply with these terms. 